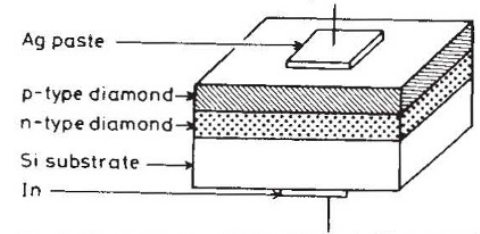

FIG. 1 Structure of the Tokai diamond diode (see text for details).

expected to result from nitrogen doping, and indeed most natural diamonds contain dissolved nitrogen, the extra electron on the nitrogen is too strongly bound and becomes ionized only at temperatures of several thousand degrees. Phosphorus has also been tried as an n-type dopant, ion implantation being used to insert it into the diamond. But the damage to the lattice caused by this technique is too great.

The Tokai work has been made possible by the advent of low-pressure techniques for making polycrystalline diamond films by chemical vapour deposition (CVD): n-type doping is then achieved by injecting diphosphorus pentoxide into the methane/hydrogen gas mixture used to form the diamond. The injection of boron trioxide allows the growth of p-type diamond, so that the same techniques can be used in diamond semiconductor fabrication as in conventional silicon technology.

The Tokai group now describe the first $\mathrm{p}-\mathrm{n}$ diode junction produced in this way $(\mathrm{K}$. Okano et al. Solid St. Elec. 34, 139-141; 1991; Appl. Phys. Lett. 58, 840-841; 1991). The diode was grown on a silicon substrate (Fig. 1) and the electrical connection was made using an indium/silver paste; melting of indium curtailed the range of operating temperatures. Furthermore the current rectification of the device (the purpose of a diode; Fig. 2) was rather poor at room temperature, owing to the high resistance of the n-type diamond. Nevertheless, after this first step, rapid progress towards an all-diamond transistor can be expected, and the question arises: what commercial applications might such devices have?

Although diamond conducts heat very efficiently, and so should make an effective heat sink, the high electrical resistivity of the doped films will impede operation at high power. Furthermore, the energy-levels of the donors and acceptors are still rather large, so the charge-carrier concentration in the diamond will be strongly temperature-dependent, unlike similarly doped silicon whose resistivity shows very little temperature

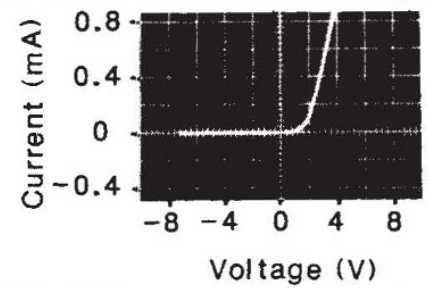

FIG. 2 Rectifying current-voltage characteristics of the Tokai diamond diode. (Both figures from Appl. Phys. Lett. 58, 840-841; 1991.) dependence over the operating range of the device. It is interesting to note that the threshold voltage for conduction in the pioneering devices reported by the group at Tokai seems to be just under $2 \mathrm{~V}$, whereas with the dopants used one might expect nearly $5 \mathrm{~V}$ for perfect diamond, which has a very large gap between the conduction and valence states. Possibly the grain boundaries in the CVD films play an appreciable role in the conduction mechanism.

The use of diamond in light-emitting diodes may also suffer drawbacks: diamond has a so-called indirect optical band gap so that lattice vibrations must become involved for light to be radiated. The high energy of the vibrations of diamond's rigid lattice makes the process inefficient, so that useful light-emitting diodes may be difficult to achieve. The most promising line, for which there is considerable demand, may well be the production of robust radiation detectors. Already such devices exist, based on Schottky barriers, and according to some reports, the high-energy-physics Superconducting Super Collider will use diamond particle detectors. This ought to provide considerable development capital on about the right timescale.

Furthermore, the civilian nuclear power industry has an urgent need for in-reactor devices which can monitor conditions such as neutron flux, or mechanical movement, under intense radiation at high temperatures. Such devices might revolutionize reactor design, making it possible to detect the incipient development of dangerous conditions with much greater ease and certainty than is now possible. Silicon devices degrade so quickly as to be almost useless, but diamond-based devices should last at least an order of magnitude longer under reactor conditions.

The first practical requirement is to produce a sufficiently thick, reproducible, highconductivity $\mathrm{p}-\mathrm{n}$ junction to permit pulse counting of particle impacts, probably on an appropriate scintillator placed before or around the junction depending upon the nature and energy of the particles to be detected. The next requirement is to perfect a system of electrical contacts compatible with operation at high temperatures. One imagines that because of the uniquely valuable information obtainable from such detectors, the necessary capital and research capability may be forthcoming to make this application of diamond-based electronics a realistic possibility within the foreseeable future.

However, whatever the future holds, the remarkable achievement by the Tokai group of an all-diamond $p-n$ junction is a milestone on the road to the production of an array of electronic devices based on a substance which in most people's minds is still just a girl's best friend.

L. M. Brown is in the Department of Physics, University of Cambridge, Madingley Road, Cambridge CB3 OHE, UK.

\section{Still sound}

MANY rubbers and plastics can 'imbibe' liquid solvents, swelling up as they do so. Curiously enough, they can also imbibe high-pressure and supercritical gases. When the pressure is released, the imbibed gas sometimes bursts its way out of the rubber - hence the enigmatic destruction of many an apparently sound gas-seal. Daedalus now plans to exploit this effect.

Consider, he says, a rubber swollen by the absorption of high-pressure gas, and then restored to atmospheric pressure. The linked network of rubber molecules is under tension; without its loading of dissolved gas it would contract. The gas molecules, by contrast, are under pressure. Without the restraint of the rubber they would expand. At some well-chosen concentration of occluded gas, the two effects should balance exactly. The material would then have no preferred volume. Over a wide density-range, it could be squashed or expanded without developing any restoring force. It would be the first totally inelastic solid.

So DREADCO engineers are pressurizing various polymers in different gases, in search of this ultimately dead product. One problem is that it will be metastable. On removal from the pressure-chamber it will immediately start to lose gas by evaporation. The best compositions, however, should hold their gas almost immobile in the polymer lattice, rather like a conventional plasticizer. Even the small amount of gas which escapes from the surface will then simply leave a skin of dense, gas-depleted polymer to impede further evaporation. The interior of the product will have zero elasticity, and will therefore transmit sound with zero velocity. In other words, it will be a perfect sound insulator.

The modern world is crying out for it. DREADCO's "Dead Silence" polymer will be rapidly adopted in engine-covers for cars, lorries and motor mowers, decor for aircraft, mufflers for pneumatic drills, screens for offices, restaurants, and telephone booths, and shielding for industrial machinery. Householders in particular will welcome it. Nearly all the world's housing was put up before, or in total disregard of, the development of the amplifier. Now, at last, Dead Silence wallpaper will bring instant relief from the most rock-crazed neighbours. The modern town house, flat, or tower-block will become at least acoustically bearable.

Even the most stable Dead Silence formulations will slowly lose gas, and shift away from perfect density-equilibrium. They will then transmit sound, but at very low velocities. You will know that redecoration is needed when you start to hear through the wall the noises your neighbours made yesterday. David Jones 